\title{
Computer digital color technology in the application of graphic design
}

\author{
Wencai Zhong \\ Environmental Management College of China, Qinhuangdao 066000, China \\ 116205@qq.com
}

Keyword: Computer digital color, Color model, Artistic design, Color application

\begin{abstract}
Developed from art, computer digital color is a new subject integrating computer technology, graphics and color science, as well as an open system which is not limited in the a certain field. In artistic design field, color model, color domain and color application rely on the five-grade color table of digital color to obtain the more precise color performance, making the original complex, disorderly and blue traditional color matching become simple and orderly with distinct levels. Computer digital color also carries out color harmony and innovation of artistic design works from several angles, such as hue, brightness, tone and color mixing, greatly promoting the development of modern color science and opening up the broad prospects of the application of digital color in the artistic design industry.
\end{abstract}

\section{Introduction}

Color is the basic element in artistic design while digital color is the new performance form of color science, which exists depending on computer digital equipments. Modern digital technology takes the advantages of various color models in the color system and constructs a color selection mode based on color attributes in the way of numerical values, thus injecting new connotations into artistic design from many aspects including medium, design method and color composition. In addition, computer digital color establishes a mapping relationship between the creation of color space and color attributes, and carries out color optimization and color management for color schemes relevant to artistic design by using the method of cluster analysis in fuzzy mathematics, thus achieving the organic connection between computer digital color and color model.

\section{Composition principle of computer digital color model}

Digital color system is composed of relevant computer color models. In computer graphics, color model is a three-dimensional color coordinate system as well as the explanation of amongst visible light subset. The purpose of using a special color model is to describe colors in a defined color domain. In general, the common color models include RGB, CMYK, HSB and Lab color model. However, in computer graphics and image software, color model is used to describe a certain color express mode, which determines the color mode of artistic design works shown in computers. Apart from the above several color modes, there are also Bitmap mode, grayscale mode, duotone mode, indexed mode, multichannel mode and 8/16 bit mode. The image description, color reproduction theory and number of color can be displayed of each mode differs from each other. These color modes are beneficial to us to accurately grasp colors in artistic design.

Analysis on RGB additive color mixture color model. RGB color model is a kind of additive color model, which produces a variety of colors through the additive mixture of the three primary colors Red, Green, and Blue in different proportions. In RGB mode, the values of 0-255 represent brightness. The higher the value of each primary color is, the brighter the color will be. When the values of R, G, and B are all 0, it is black after mixture; and when the values are all 255, it is white. If the three primary colors Red, Green, and Blue are distributed in each level in accordance with the brightness value from 0 to 255, there will be about 16.7 million colors. We describe the RGB color model with the cube in Cartesian rectangular coordinate system, as shown in Fig. 1. The horizontal $\mathrm{X}$ axis represents red, which increases to the right; $\mathrm{Y}$ axis represents blue, which increases to the left bottom; the vertical $\mathrm{Z}$ axis represents green, which increases upward; the base point represents 
black, which is hidden in the back of the cube. RGB color model is the basis for computer digital equipments to display colors, as well as the most typical and commonly used color model of computer digital color.

CMYK subtractive color mixture color model. CMYK color model is a kind of printing mode composed of cyan, magenta, yellow and black. It represents the change of the purity of each color with the values from 0 to 100 . in practice, due to the chemical characteristics of color ink and printing ink in color printing and multicolor printing, as well as the factors such as the light reflection and the paper's adsorption degree of colors, we can't obtain black through equal mixing of the CMY primary colors. Therefore, black needs to be added in to make up for the deficiency. From Cartesian rectangular coordinate system, it is obvious that the appearance of CMYK color model is similar to RGB color model, but their base points and vertex are exactly the opposite. The base point of CMYK model is white while the vertex is black. Unlike RGB model which adds a certain color into black light, CMYK model refers to subtract a certain color from white light. While mixing $\mathrm{C}, \mathrm{M}, \mathrm{Y}$, and $\mathrm{K}$, the brightness of the light will be lower with the increasing of the four colors. Therefore, CMYK mode is also called subtractive color process.
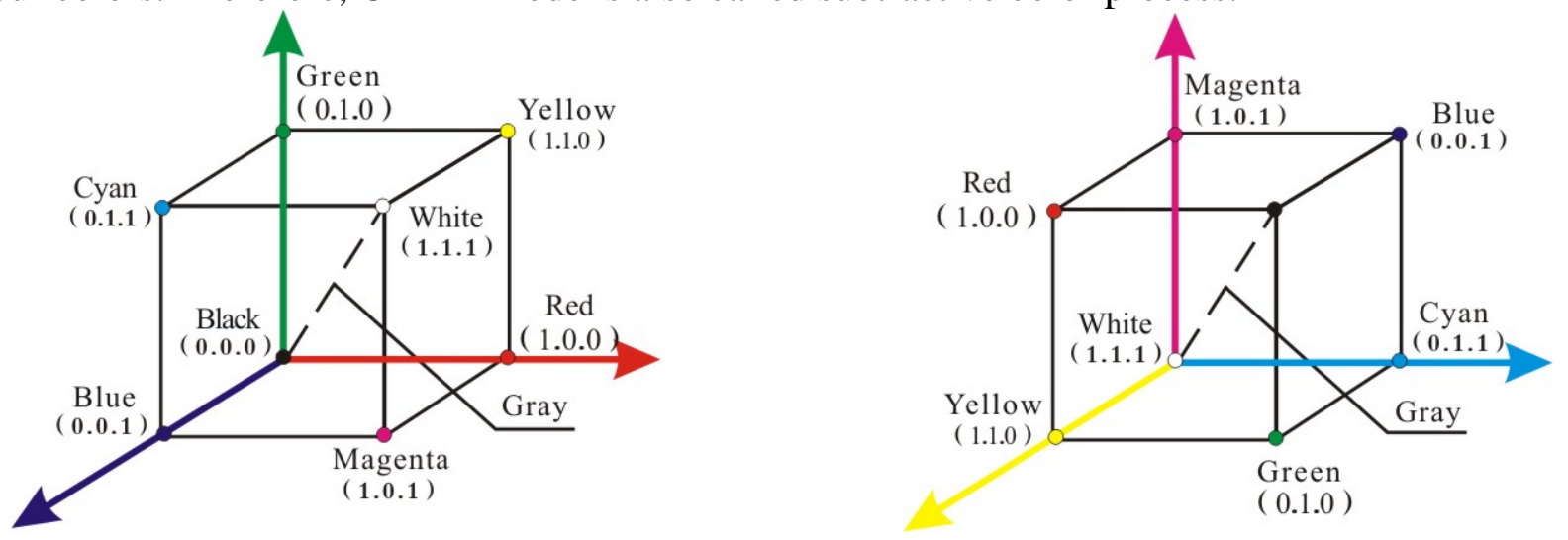

Fig. 1 RGB three-dimensional color model in Cartesian coordinate

Intuitive color model HSB. HSB color model is evolved from RGB, which adopts the user intuitive color description method. It is defined on basis of human observation of colors. All the colors are represented with hue, saturation and brightness. HSB color model is often used in computer graphics applications. In artistic design, usually HSB color wheel is used to select a color for a specific application environment of graphic elements. In the HSB color wheel (Fig. 2), hue represents a pure colour in the hue circle. In the 0-360 degrees standard color wheel, hue is measured according to the location. a separate triangle is used to show the saturation and brightness. The vertical axis of this triangle indicates the saturation, representing the percentage of the pure color. When $S=1$, the purity of the color is the highest; when $S=0$, it shows the gray-scale value. The horizontal axis indicates the brightness, representing the brightness of the color. When $\mathrm{B}=0$, it shows black. In this way, while applying HSB color model in artistic design, we can first choose a hue in the hue circle and then choose the desired saturation and brightness in the triangle, thus eliminating the communication obstacle between digital colors and traditional paint colors.
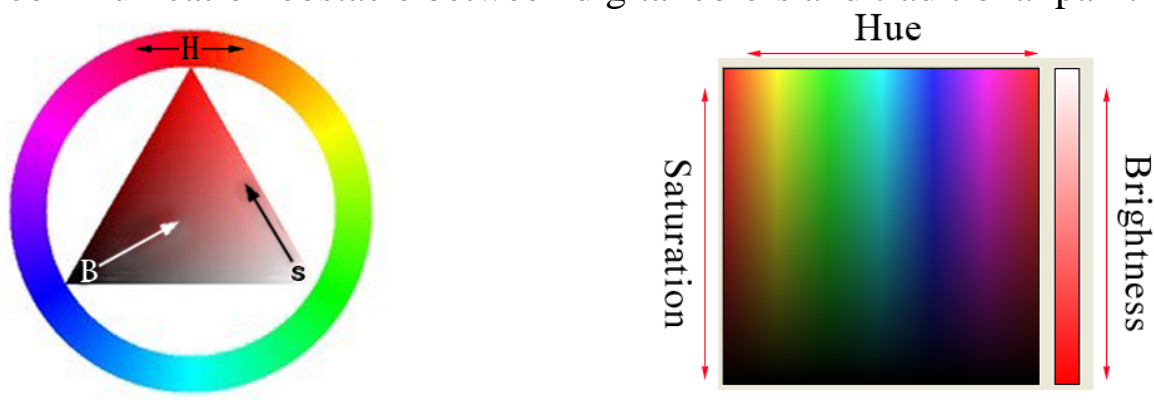

Fig. 2 HSB color model 
Lab color model independent on devices. Lab color model is composed of three elements, L, a, and b. Here, L represents Luminosity, equivalent to luminance; a represents the scope from red to green; b represents the scope from blue to yellow. Due to the characteristics of wide color gamut, Lab color model not only contains all the color gamut of RGB and CMYK, showing the colors which they fail to display, but also makes up for the problems of uneven color distribution and color deficiency of RGB color model. It is because that in RGB model, there are too many intermediate colors between blue and green while yellow and other colors are in lack between green and red. Therefore, in order to retain rich colors as possible in the processing of digital graphics, it's better to choose Lab color model. In addition, Lab describes the display mode of color, rather than the amount of specific colors needed by the device (monitor, printer or digital camera) to generate the color. It can exchange graphics colors in different computer systems, and avoid the color difference caused by different computer printing devices, thus keeping the graphics and colors consistent. As a result, Lab is also regarded as a color model independent on devices.

The composition of computer digital color model is based on modern color science and computer graphics. It adopts the color analysis of artistic color science and forms unique color area, change rules and technical standards. It is because of such scientific and overall structure that digital color can endow more diversified design means to artistic design.

\section{Digital color mixing forms in artistic design}

Digital color means to express color with digital. Its mixing form refers to carry out color mixing through the number of digitals used to express colors, or the relevant elements. The production of digital color mixing form is based on hand-painted colors. Based on Munsell's color theory, actual toning is carried out in proportion according to the principle of digital quantization. According to the define factors of digital color mixing forms, it can fully show the chromatic colors mutual mixing, neutral colors mutual mixing, mutual mixing of chromatic colors and neutral colors, mixing of neutral colors added in mutual mixing of chromatic colors. For example, in plane advertisement design, Photoshop is used to mix two different colors, thus forming the third composite color. Such mixing can be either the combination of secondary color and secondary color or the combination of primary color and secondary color. Through the equal mixing of the three primary colors and the three secondary colors, we can obtain yellow orange, red orange, red purple, blue purple, blue green, and yellow green. Composite colors contain all the colors except the primary and secondary colors. According to the above color mixing categories, the digital color combinations are listed in Table 1.

Table 1 Diagram of digital color mixing forms

\begin{tabular}{l|l|l}
\hline Color mixing recognition & \multicolumn{1}{|c|}{ Color mixing forms } & \multicolumn{1}{c}{ Color expression explanation } \\
\hline \multirow{2}{*}{$\begin{array}{l}\text { Mixing of chromatic } \\
\text { colors }\end{array}$} & $5 \mathrm{R} \cdot 5 \mathrm{G} / 0$ & $5 \mathrm{Red}+5$ Green \\
\cline { 2 - 3 } & $6 \mathrm{R} \cdot 4 \mathrm{Y} / 0$ & 5 Red+4 Yellow \\
\hline \multirow{2}{*}{ Mixing of neutral colors } & $0 / 5 \mathrm{~W} \cdot 5 \mathrm{~B}=0 / 10 \mathrm{~N} 5$ & $5 \mathrm{White}+5$ Black=No.5 Grey \\
\cline { 2 - 3 } & $0 / 9 \mathrm{~W} \cdot 1 \mathrm{~B}=0 / 10 \mathrm{~N} 1$ & 9 White+1 Black=No.1 Grey \\
\hline \multirow{2}{*}{$\begin{array}{l}\text { Mixing of chromatic } \\
\text { color and neutral color }\end{array}$} & $5 \mathrm{R} / 5 \mathrm{~W}$ & 5 Red +5 White \\
\cline { 2 - 3 } & $6 \mathrm{G} / 4 \mathrm{~B}$ & 6 Green +4 Black \\
\cline { 2 - 3 } $\begin{array}{l}\text { Mixing of neutral color } \\
\text { added in mixing of } \\
\text { chromatic colors }\end{array}$ & $5 \mathrm{R} \cdot 3 \mathrm{G} / 2 \mathrm{~W}$ & 7 Red +3 No.1 Grey \\
\cline { 2 - 3 } & $6 \mathrm{G} \cdot 2 \mathrm{P} / 2 \mathrm{~B}$ & 5 Red +3 Green +2 White \\
\hline
\end{tabular}

Through understanding the mixing principle of digital colors, it is clear that most of the colors in artistic design are formed after mutual mixing, which helps us to get a certain understanding of the rules of color matching. In design practice, we can make color matching more precise and highlight the particularly important role of digital color in artistic design. 


\section{Application forms of digital color in artistic design}

In the field of artistic design, design works generally have a variety of colors, so as to obtain a sense of harmony and unification of the overall color combination. Most applications of digital color will select a color as the main tone of the picture according to the themes of the works and the needs of visual appeal. After determining the main color tone, choose the color which occupies the largest area in the picture, and then obtain the overall tone with different emotional tendencies in accordance with the principles of color combination, including the combination of hue, brightness and purity.

Digital color has three performance forms of repetition, alternation and gradation. In artistic design, "repetition" means to produce a inflective color tone with kinetic potential and constitute a rich and orderly visual effect through the continuous appearance of the hue, brightness, purity and strength change of the a same color; "alternation" means the continuous appearance of a same color. Different from repetition, other colors will be inserted into the appearances of the same color as intervals. It can stimulate the audiences vision repeatedly with certain intervals; "gradation" is also called gradient, which refers to the inconsecutive sequence change with multicolor combination in artistic design, showing a state of gradual change of color, such as the changes in light and shade, strength, and depth. Such changes can be the gradient of the hue levels, or the changes in color brightness and purity.

When in face of an artistic design and want to reach a good visual effect, we should not only focus on innovation, graphics and text, and layout, but also pay attention to the application and performance of digital color. In this aspect, we should emphasize on the role of color emotion rules in artistic design, as well as the application of color combination in design. Only these two aspects are well done can we best express a good artistic design.

\section{Conclusion}

As a carrier of information, color is applied in artistic design in the form of digital, reproducing the importance of the multi disciplinary integration of computer digital technology, color science and design aesthetics. Research on the composition of color model can be carried out through the application software used to design colors, scientific design and arrangement of color matching, making its application significance more prominent in artistic design. It is the scientific and overall structure of color design that leads to the more precise color performance in artistic design, thus reproducing the charm of digital color.

\section{References}

[1] S.X.Tian,L.Tan:Digital Color, (Central Radio and Television University Publishing House, In Chinese 2010)

[2] Y.L.Xiao : Fundamentals of digital color, (Beijing Normal University Press, In Chinese 2008)

[3] T.Hu:Theory and Application of Computer Color, (Tsinghua University Press, In Chinese 2014)

[4] P. Zhaoeng:Detailed Explanation on the Color Mode in Computer Artistic Design,( http:// www.zhaopeng.net) 